\title{
Clinical utility of urinary levels of catecholamines and their fraction ratios related to heart rate and thyroid function
}

\author{
Naruhiko Sunada, Yoshihisa Hanayama, Koichiro Yamamoto, Yasuhiro Nakano, Takahiro Nada, \\ Hiroyuki Honda, Kou Hasegawa, Hideharu Hagiya and Fumio Otsuka
}

Department of General Medicine, Okayama University Graduate School of Medicine, Dentistry and Pharmaceutical Sciences, Okayama 700-8558, Japan

\begin{abstract}
Urinary catecholamines (CAs) have been examined for the screening of pheochromocytomas. The decision to perform screening is based on symptoms suggesting secondary hypertension or hyperactivities of the sympathetic nervous system. To elucidate the usefulness of urinary fractions and ratios of CAs, 79 patients in whom 24-h excretions of urinary CAs including adrenaline (AD), noradrenaline (NA) and dopamine (DA) had been examined from 2015 until 2020 were retrospectively analyzed. There were no significant differences in urinary CA levels between two age groups, gender groups and two BMI groups. Patients with histories of preexisting hypertension and diabetes showed significantly higher levels of urinary NA excretion, and the urinary ratio of NA/DA was also increased in the patients with a history of hypertension. Heart rate (HR) was significantly correlated with the urinary ratio of NA/DA. Serum free thyroxine (FT4) concentration and ratio of FT4/thyrotropin (TSH) were correlated with the level of urinary AD. The levels of TSH and FT4/TSH showed negative and positive correlations, respectively, with the urinary NA/DA ratio. Thus, increases of HR are related to the enhanced conversion of DA to NA and increased thyroid hormones are involved in the increase in urinary AD and the conversion of DA to NA. History of lifestyle-related diseases and changes of HR and thyroid functions need to be considered for the evaluation of urinary CAs and their ratios.
\end{abstract}

Key words: Adrenaline, Catecholamine, Hypertension, Noradrenaline, Thyroid function

LEVELS of catecholamines (CAs) in plasma and urine are often examined to evaluate the possibility of pheochromocytomas and/or paragangliomas (PPGLs) in hypertensive patients [1]. The patients with PPGLs have various symptoms: so-called "5-H" symptoms that arise from tumor-producing CAs including hypertension (HT), headaches, hyperhidrosis, hypermetabolism and hyperglycemia [2-4]. Therefore, plasma and/or urinary levels

Submitted Aug. 3, 2021; Accepted Oct. 12, 2021 as EJ21-0488

Released online in J-STAGE as advance publication Nov. 3, 2021

Correspondence to: Fumio Otsuka, M.D. Ph.D., Department of General Medicine, Okayama University Graduate School of Medicine, Dentistry and Pharmaceutical Sciences, 2-5-1 Shikatacho, Kita-ku, Okayama 700-8558, Japan.

E-mail: fumiotsu@md.okayama-u.ac.jp

Abbreviations: AD, adrenaline; BMI, body mass index; CA, catecholamine; CKD, chronic kidney disease; DA, dopamine; DOPA, dihydroxyphenylalanine; DBP, diastolic blood pressure; HT, hypertension; HR, heart rate; NA, noradrenaline; FT4, free thyroxine; PNMT, phenylethanolamine N-methyltransferase; PPGLs, pheochromocytomas and/or paragangliomas; SBP, systolic blood pressure; TSH, thyrotropin of CAs are often examined in patients with such symptoms. Other symptoms in patients with PPGLs include anxiety, nausea, vomiting, and weakness [5].

CAs include dopamine (DA), noradrenaline (NA), and adrenaline (AD), which are synthesized from tyrosine. Tyrosine hydroxylase (TH) converts tyrosine to 3,4dihydroxyphenylalanine (DOPA), and this is followed by conversion by DOPA decarboxylase of DOPA to DA, conversion by dopamine $\beta$-hydroxylase of DA to $\mathrm{NA}$, and conversion by phenylethanolamine $\mathrm{N}-$ methyltransferase (PNMT) of NA to AD [6-8]. In this cascadic process, the action of $\mathrm{TH}$ is considered to be a rate-limiting step [9]. In clinical situations, plasma CA concentrations and urinary levels of CA excretion are often utilized to determine the systemic secretory activities of CAs that are produced by the adrenal medulla and various sympathetic ganglions. The biochemical diagnosis of PPGLs consists of the demonstration of hypersecretion of CAs or their O-methylated metabolites, the latter of which show a high sensitivity for detecting PPGLs [10]. 
However, the clinical usefulness and significance of measurements of urinary CAs other than for examining the possibilities of PPGLs have not been established. In the present study, we retrospectively investigated the clinical significance of urinary excretions of CAs and the relative ratios of $\mathrm{CA}$ fractions.

\section{Patients and Methods}

\section{Study design}

We retrospectively screened the medical records of 79 patients whose levels of urinary CAs were examined during the period from January 2015 to December in 2020 at the Department of General Medicine, Okayama University Hospital. Of those patients, 5 patients with active PPGLs, 1 patient with adrenal hyperplasia, 1 patient with adrenal lymphoma and 6 patients who had been prescribed catecholaminergic agonists were excluded. As a result, 66 patients ( 27 males, 39 females) were included in the present analysis. Examination of urinary CAs was decided by each physician when hyperor hypo-secretion of CAs was clinically suspected on the basis of each patient's symptoms, laboratory data, and past medical history. All of the urinary collections for CAs excretions were performed in hospitalized conditions for one to two days in our hospital. Data for other biochemical markers were also obtained within a few days of measurement of urinary CA excretions. These examinations were performed for the patients under adequate insurance coverage. Information regarding the present study was provided on our hospital wall and on the website of our hospital, and patients who wished to opt out were offered that opportunity. This study was approved by the Ethical Committee of Okayama University Hospital (KEN 2106-016) and adhered to the Declaration of Helsinki.

\section{Analysis of clinical parameters}

Information on the patients' chief complaints was obtained from hospital medical records. Data on age, gender, body mass index (BMI), self-rated depression scale (SDS), and frequency scale for symptoms of gastroesophageal reflux disease (FSSG) were also obtained. Diagnosis of hypertension was based on the definition of hypertension: having a systolic blood pressure (SBP) $\geq 140 \mathrm{mmHg}$, having a diastolic blood pressure (DBP) $\geq 90 \mathrm{mmHg}$, or taking BP lowering agents [11]. Values for the following blood biochemical parameters were obtained from records, when available: white blood cells (WBC), hemoglobin, and platelets for blood cell counts; total bilirubin (T.Bil), albumin, aspartate aminotransferase (AST), alanine aminotransferase (ALT), alkaline phosphatase (ALP), lactate dehydrogenase (LDH), creatinine $(\mathrm{Cr})$, urea nitrogen (UN), estimated glomerular filtration rate (eGFR), sodium, potassium, and corrected calcium (Ca) for liver and renal functions; blood glucose, hemoglobin A1c (HbA1c), low-density lipoprotein cholesterol (LDL-C) and high-density lipoprotein cholesterol (HDL-C) as metabolic markers; and thyrotropin (TSH), free thyroxine (FT4), free triiodothyronine (FT3), and ratio of TSH/FT4 as thyroid function markers. eGFR was calculated by using the following formulas: $194 \times$ $\mathrm{Age}^{-0.287} \times \mathrm{Cr}^{-1.094}$ for males and $194 \times \mathrm{Age}^{-0.287} \times$ $\mathrm{Cr}^{-1.094} \times 0.739$ for females. Urinary CA levels were determined by high-performance liquid chromatography (HPLC; BML, Inc., Saitama, Japan) in 24-h collected urine samples with 6-M hydrochloric acid to prevent degradation of collected urinary CAs [12]. The normal ranges of urinary $\mathrm{CAs}$ are as follows: $\mathrm{AD}$ : $3.0-$ $41.0 \mu \mathrm{g} / \mathrm{day}$; NA: 31.0-160.0 $\mu \mathrm{g} /$ day; and DA: $280.0-$ $1,100.0 \mu \mathrm{g} /$ day. All other levels were determined using an auto-analyzer system at the Central Laboratory of Okayama University Hospital.

\section{Statistical analysis}

EZR, version 1.40 (Saitama Medical Center, Jichi Medical University, Saitama, Japan), which is a graphical user interface for R (The R Foundation for Statistical Computing, Vienna, Austria), was used in all statistical analyses [13]. It is modified from $\mathrm{R}$ commander, which is designed to add frequently used functions in biostatistics. The Mann-Whitney $U$ test or Spearman's rank correlation coefficient was used to statistically analyze continuous measurements. All tests were performed as two-sided, and $p<0.05$ was regarded as statistically significant.

\section{Results}

The mean age of the patients was 64 years (interquartile range: IQR, 45-69 years), the male/female ratio was $39(59 \%) / 27(41 \%)$, and mean BMI was $24.1 \mathrm{~kg} / \mathrm{m}^{2}$ (IQR, 21.6-26.0 kg/m²). As shown in Fig. 1, it was found that there were no significant differences in urinary levels of $\mathrm{AD}, \mathrm{NA}$ and $\mathrm{DA}$ or the ratios of $\mathrm{AD} / \mathrm{NA}$ and NA/DA between the two age groups $(<65$ vs. $\geq 65$ years), two gender groups (M vs. F) and two BMI groups $\left(<25 v s . \geq 25 \mathrm{~kg} / \mathrm{m}^{2}\right)$. In patients with urinary NA higher than the normal upper limit ( $>160 \mu \mathrm{g} /$ day), 52\%, 61\% and $45 \%$ of the patients were older patients ( $\geq 65$ years), female patients and patients with high BMI $\left(\geq 25 \mathrm{~kg} / \mathrm{m}^{2}\right)$, respectively, whereas $46 \%, 57 \%$ and $34 \%$ (slightly lower percentages) of the patients with normal urinary NA ( $\leq 160 \mu \mathrm{g} /$ day) were older patients $(\geq 65$ years), female patients and patients with high BMI $\left(\geq 25 \mathrm{~kg} / \mathrm{m}^{2}\right)$, respectively. 
A
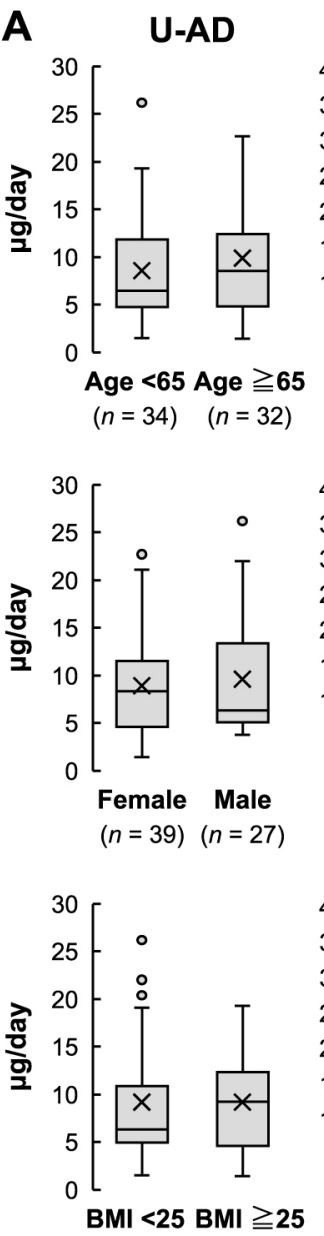

$(n=40) \quad(n=26)$
U-NA
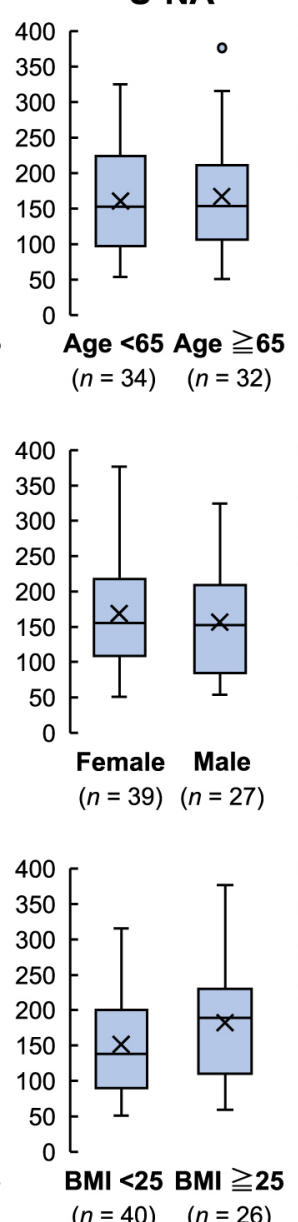

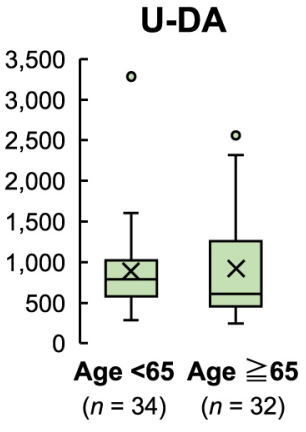

B
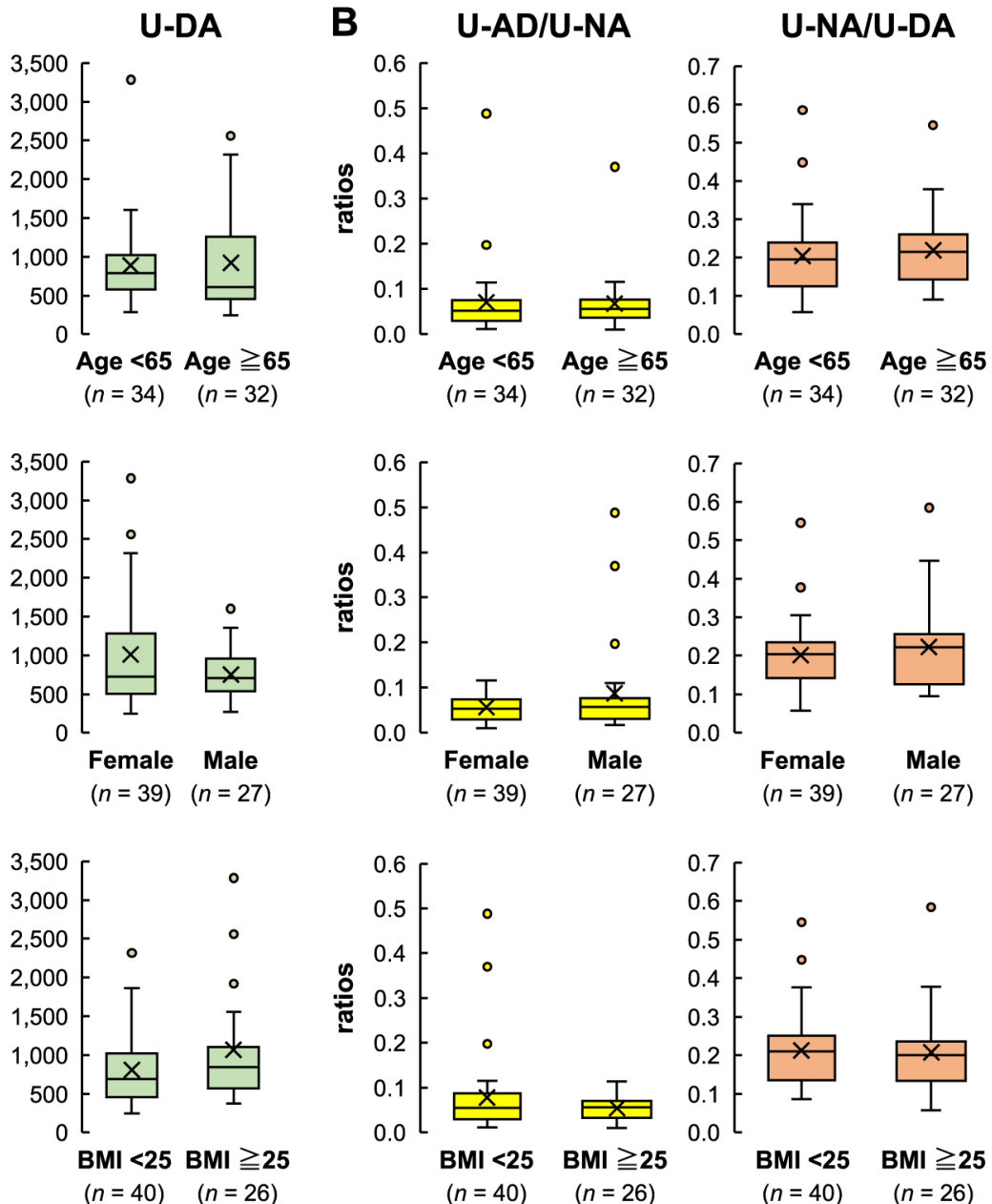
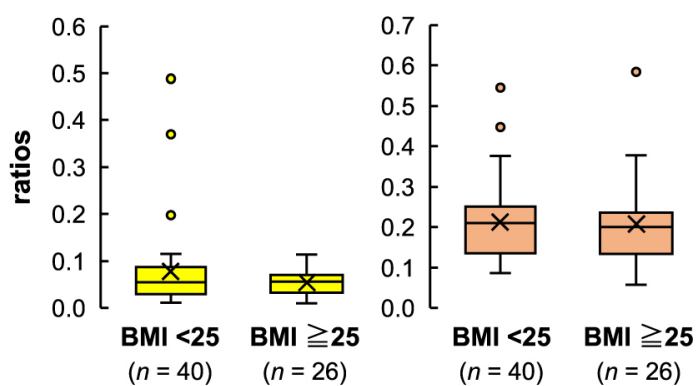

Fig. 1 Urinary levels of catecholamines and their fraction ratios based on the characteristics of patients in the present study. Urinary levels of all catecholamines (CAs; A) including adrenaline (AD), noradrenaline (NA) and dopamine (DA) and the urinary ratios (B) were compared in two age groups ( $<65 v s$. $\geq 65$ years), two gender groups (male $v s$. female), and two body mass index (BMI) groups ( $<25 \mathrm{vs} . \geq 25 \mathrm{~kg} / \mathrm{m}^{2}$ ). In each panel, the upper horizontal line of the box is the 75 th percentile, the lower horizontal line of the box is the 25th percentile, the horizontal bar within the box is the median, the upper horizontal bar outside the box is the maximum value within 1.5 times the interquartile range, and the lower horizontal bar outside the box is the minimum value within 1.5 times the interquartile range.

The mean values of SBP, DBP and heart rate (HR) were $129 \mathrm{mmHg}(\mathrm{IQR}, 119-144 \mathrm{mmHg}), 78 \mathrm{mmHg}(75-$ $85 \mathrm{mmHg}$ ) and $74 \mathrm{bpm}(64-78 \mathrm{bpm})$, respectively. The major symptoms for which it was decided to perform CA measurements were headaches in 13 cases (19.7\%), palpitation in 8 cases $(12.1 \%)$, hyperhidrosis in 6 cases (9.1\%), and body weight loss in 6 cases $(9.1 \%)$. The medical histories of the patients included HT in 53 patients $(80.3 \%)$, glucose intolerance $(\mathrm{HbA} 1 \mathrm{c} \geq 6.5)$ in 8 patients $(12.1 \%)$, and chronic kidney diseases (CKD) in 12 patients $(18.2 \%)$.

Next, the impacts of clinical backgrounds including histories of HT, CKD and diabetes mellitus were assessed in relation to the levels of urinary levels of $\mathrm{AD}$, $\mathrm{NA}$ and DA or the ratios of $\mathrm{AD} / \mathrm{NA}$ and NA/DA. As shown in Fig. 2, among the medical histories of HT,
CKD and diabetes mellitus, patients with preexisting HT and diabetes (hemoglobin A1c $\geq 6.5$ ) showed significantly higher levels of urinary NA excretion. In patients with urinary NA higher than the normal upper limit (>160 $\mu \mathrm{g} /$ day), $90 \%, 20 \%$ and $20 \%$ of the patients had preexisting HT, CKD and diabetes, respectively, whereas $71 \%, 17 \%$ and $7 \%$ (slightly lower percentages) of the patients with normal urinary NA $(\leq 160 \mu \mathrm{g} /$ day $)$ had HT, CKD and diabetes, respectively. Of interest, urinary ratios of $\mathrm{AD} / \mathrm{NA}$ were significantly lower in the patients with medical histories of HT and diabetes than in the patients without these disorders. On the other hand, urinary ratio of NA/DA in the patients with a history of HT was significantly higher than that in the patients without a history of HT, suggesting that the conversion step from DA to NA could be clinically linked to the etiology of HT. 
A
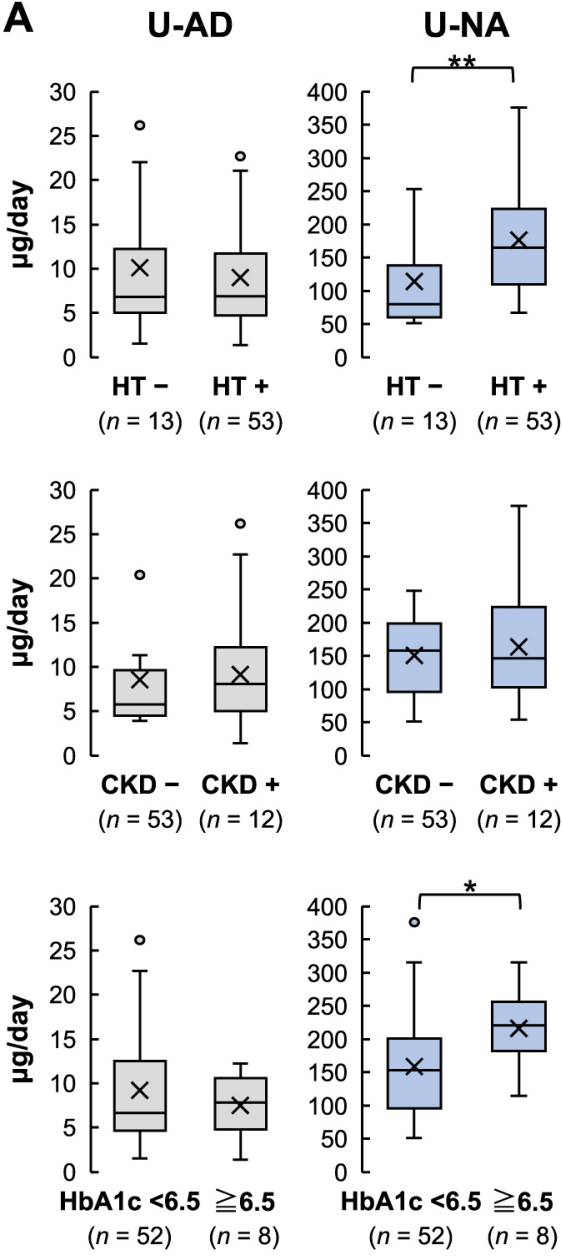

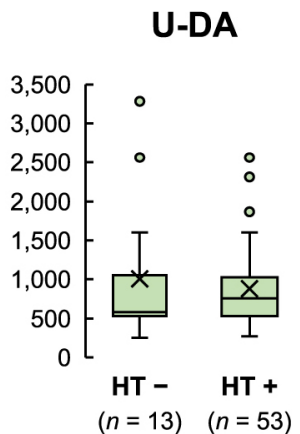

B

U-AD/U-NA

U-NA/U-DA
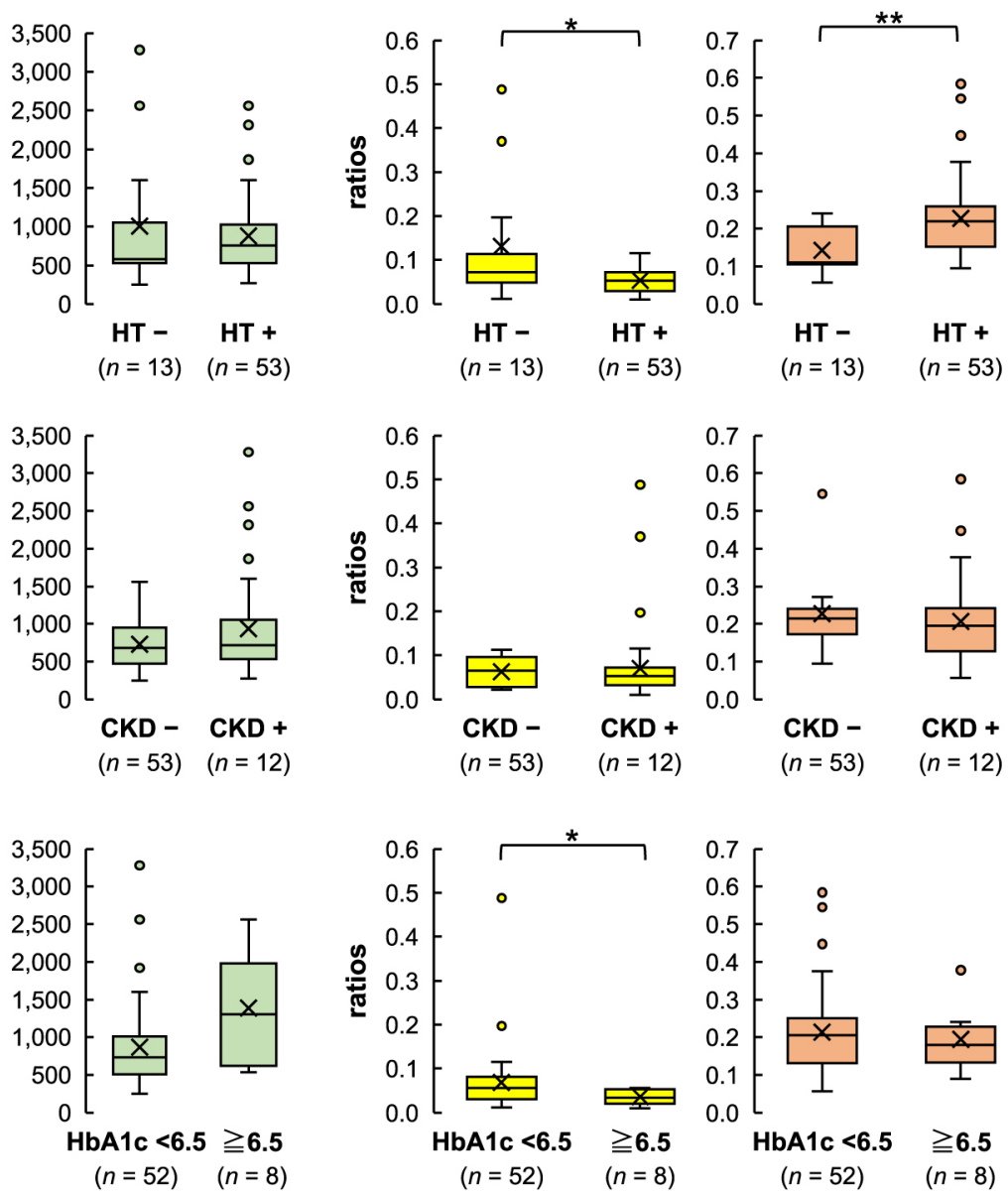

Fig. 2 Urinary levels of catecholamines and their fraction ratios based on the clinical background of patients in the present study. Urinary levels of all catecholamines (CAs; A) including adrenaline (AD), noradrenaline (NA) dopamine (DA) and the urinary ratios (B) were compared in two groups based on the patients' medical background including hypertension (HT) $-v s$. HT +, chronic kidney diseases $(\mathrm{CKD})-v s . \mathrm{CKD}+$, and diabetes mellitus: hemoglobin A1c $(\mathrm{HbA} 1 \mathrm{c})<6.5 v s . \mathrm{HbA} 1 \mathrm{c} \geq 6.5$. Bar graphs in each panel were made as described in the legend of Fig. $1 .{ }^{* *} p<0.01$ and $* p<0.05$, statistically significant differences between the indicated groups.

The relationships of various clinical data with urinary levels of CAs and ratios of CAs are shown in Table 1. Considering the process of the production of CAs, we also focused on the enzymatic conversion steps from DA to NA and the following step caused by PNMT from NA to $\mathrm{AD}$. To characterize the converting activities in the adrenal medulla and sympathetic ganglia, urinary ratios of AD/NA and NA/DA were calculated and statistically analyzed. It was found that urinary ratios of NA/DA were significantly correlated to HR, serum LDL-C levels and thyroid functions. In addition, blood glucose levels were positively correlated with urinary levels of NA but negatively correlated with urinary ratios of $\mathrm{AD} / \mathrm{NA}$, whereas $\mathrm{HbA}$ 1c levels were not significantly correlated with either urinary level of CAs or the ratios.

The key clinical symptoms related to the actions of CAs were compared to the urinary levels of CAs. As shown in Fig. 3A, individual levels of SBP were not significantly correlated with either urinary level of $\mathrm{AD}$ or NA. On the other hand, HR was significantly ( $r=0.285$; $p<0.05)$ correlated with the ratio of urinary levels of NA/DA (Fig. 3B).

Furthermore, to try to determine the factors involved in the regulation of $\mathrm{HR}$, results of thyroid function tests in relation to changes of HR were analyzed. As shown in Fig. 4, it was notable that serum FT4 concentration $(r=$ $0.443 ; p<0.01$; Fig. $4 \mathrm{~A})$ and ratio of serum FT4/TSH $(r$ $=0.291 ; p<0.05$; Fig. 4C) were significantly correlated with the level of urinary AD excretion. The level of serum TSH $(r=-0.344 ; p<0.05$; Fig. 4B) and ratio of serum FT4/TSH $(r=0.373 ; p<0.01$; Fig. 4C) showed significant negative and positive correlations, respectively, with the ratio of urinary levels of NA and DA. 
Table 1 Relationships of clinical data with urinary levels of catecholamines and their ratios

\begin{tabular}{|c|c|c|c|c|c|c|c|c|c|}
\hline \multirow{2}{*}{ CA, Ratios } & \multicolumn{2}{|c|}{ U-AD } & \multicolumn{2}{|c|}{ U-NA } & \multicolumn{2}{|c|}{ U-AD/NA } & \multicolumn{2}{|c|}{ U-NA/DA } & \multirow{2}{*}{$N$} \\
\hline & $r$ & $p$ values & $r$ & $p$ values & $r$ & $p$ values & $r$ & $p$ values & \\
\hline \multicolumn{10}{|c|}{ Patients' profile } \\
\hline Age & 0.0559 & 0.656 & 0.0949 & 0.449 & -0.0236 & 0.851 & 0.201 & 0.105 & 66 \\
\hline BMI & 0.000773 & 0.995 & $\underline{0.26}$ & $\underline{0.0349 *}$ & -0.18 & 0.148 & -0.0507 & 0.686 & 66 \\
\hline SDS & 0.34 & 0.104 & -0.0262 & 0.903 & 0.252 & 0.234 & 0.018 & 0.934 & 24 \\
\hline FSSG & -0.322 & 0.134 & -0.147 & 0.504 & -0.0367 & 0.868 & 0.065 & 0.768 & 23 \\
\hline SBP & -0.189 & 0.128 & -0.0832 & 0.506 & -0.112 & 0.372 & 0.0503 & 0.688 & 66 \\
\hline HR & 0.0447 & 0.722 & 0.231 & 0.0618 & -0.152 & 0.224 & $\underline{0.285}$ & $\underline{0.0205^{*}}$ & 66 \\
\hline \multicolumn{10}{|c|}{ Laboratory data } \\
\hline WBC & -0.00355 & 0.978 & 0.207 & 0.101 & -0.0759 & 0.551 & -0.0134 & 0.916 & 64 \\
\hline Hemoglobin & 0.0732 & 0.565 & 0.161 & 0.204 & -0.101 & 0.427 & -0.0501 & 0.694 & 64 \\
\hline Platelets & 0.101 & 0.428 & $\underline{0.248}$ & $\underline{0.0479 *}$ & -0.00365 & 0.977 & 0.0689 & 0.589 & 64 \\
\hline T. Bil & -0.0713 & 0.591 & -0.215 & 0.102 & 0.0414 & 0.756 & -0.0318 & 0.811 & 59 \\
\hline Albumin & 0.0747 & 0.558 & -0.116 & 0.36 & 0.0734 & 0.564 & -0.0922 & 0.469 & 64 \\
\hline $\mathrm{AST}$ & -0.128 & 0.318 & 0.219 & 0.0847 & -0.239 & 0.0594 & 0.0291 & 0.821 & 63 \\
\hline ALT & -0.104 & 0.408 & 0.225 & 0.0717 & -0.274 & $\underline{0.0274 *}$ & -0.0663 & 0.6 & 65 \\
\hline ALP & -0.163 & 0.201 & 0.0597 & 0.642 & -0.096 & 0.454 & 0.197 & 0.121 & 63 \\
\hline LDH & 0.0141 & 0.913 & 0.219 & 0.0852 & -0.116 & 0.366 & 0.0335 & 0.795 & 63 \\
\hline Creatinine & 0.00554 & 0.965 & -0.00674 & 0.958 & 0.0681 & 0.59 & 0.173 & 0.168 & 65 \\
\hline Urea nitrogen & -0.0399 & 0.752 & 0.145 & 0.25 & -0.121 & 0.337 & 0.103 & 0.414 & 65 \\
\hline eGFR & -0.0165 & 0.896 & -0.0925 & 0.463 & -0.0217 & 0.864 & -0.195 & 0.12 & 65 \\
\hline Sodium & -0.0721 & 0.568 & -0.00298 & 0.981 & -0.0974 & 0.44 & -0.0699 & 0.58 & 65 \\
\hline Potassium & 0.069 & 0.585 & 0.14 & 0.266 & -0.0558 & 0.659 & -0.0229 & 0.856 & 65 \\
\hline Corrected Ca & 0.2 & 0.112 & 0.163 & 0.197 & 0.0544 & 0.67 & -0.000289 & 0.998 & 64 \\
\hline \multicolumn{10}{|c|}{ Endocrine and metabolic data } \\
\hline LDL-C & 0.057 & 0.679 & 0.0905 & 0.511 & -0.0224 & 0.871 & -0.278 & $\underline{0.0402 *}$ & 55 \\
\hline HDL-C & 0.0448 & 0.752 & 0.0802 & 0.572 & -0.0696 & 0.624 & 0.149 & 0.291 & 52 \\
\hline Glucose & 0.052 & 0.706 & $\underline{0.551}$ & $\underline{0.0000131 * *}$ & $\underline{-0.355}$ & $\underline{0.00787 * *}$ & 0.229 & 0.093 & 55 \\
\hline $\mathrm{HbA1c}$ & -0.0437 & 0.74 & 0.22 & 0.0906 & -0.223 & 0.0867 & 0.0469 & 0.722 & 60 \\
\hline TSH & -0.211 & 0.125 & -0.0983 & 0.479 & -0.147 & 0.289 & -0.344 & $\underline{0.0108^{*}}$ & 54 \\
\hline FT3 & 0.0307 & 0.901 & -0.193 & 0.427 & 0.0894 & 0.716 & -0.105 & 0.67 & 19 \\
\hline FT4 & $\underline{0.443}$ & $\underline{0.000896 * *}$ & 0.162 & 0.248 & 0.222 & 0.109 & 0.205 & 0.141 & 53 \\
\hline FT4/TSH & $\underline{0.291}$ & $\underline{0.0347^{*}}$ & 0.109 & 0.437 & 0.202 & 0.146 & $\underline{0.373}$ & $\underline{0.00592 * *}$ & 53 \\
\hline BNP & 0.216 & 0.31 & 0.0805 & 0.709 & 0.112 & 0.602 & -0.207 & 0.332 & 24 \\
\hline
\end{tabular}

** $p<0.01$ and $* p<0.05$ between the indicated factors (data underlined). ALP, alkaline phosphatase; ALT, alanine aminotransferase; AST, aspartate aminotransferase; BMI, body mass index; BNP, brain natriuretic peptide; Ca, calcium; eGFR, estimated glomerular filtration rate; FSSG, frequency scale for symptoms of gastroesophageal reflux disease; FT3, free triiodothyronine; FT4, free thyroxine; HbA1c, hemoglobin A1c; HDL-C, high-density lipoprotein cholesterol; HR, heart rate; LDH, lactate dehydrogenase; LDL-C, low-density lipoprotein cholesterol; SBP, systolic blood pressure; SDS, self-rating depression scale; T. Bil, total bilirubin; TSH, thyrotropin and WBC, white blood cells.

\section{Discussion}

In the present study, urinary NA levels were found to be increased in patients with histories of HT and diabetes, in which the conversion step from DA to NA is likely to be involved in the medical history of HT. Of note, it was revealed that increases of HR are related to enhancement of the conversion of urinary DA to NA and that increased thyroid hormones are potentially involved in the increase in urinary $\mathrm{AD}$ and the conversion of $\mathrm{DA}$ to NA.

As a background of the patients, patients with preexisting HT showed significantly higher levels of urinary NA excretion and urinary ratios of NA/DA. In this 

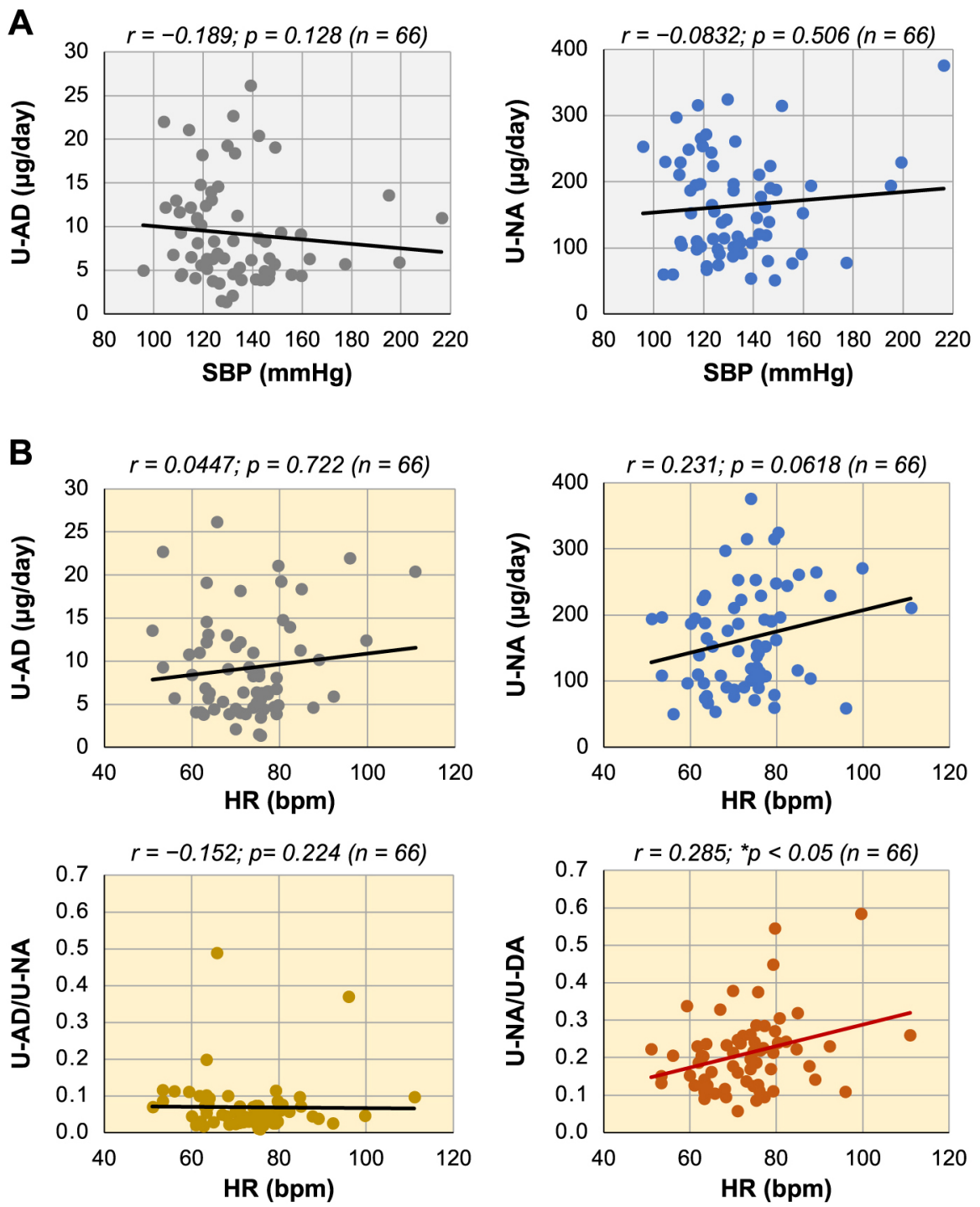

Fig. 3 Interrelationships between the levels of blood pressure, heart rate and urinary catecholamines. The patients were further examined for interrelationships between urinary levels of catecholamines (CAs), including adrenaline (AD) and noradrenaline (NA), and levels of systolic blood pressure (SBP; A). The interrelationships between urinary levels of CAs or ratios of urinary AD/NA and NA/DA and heart rate (HR; B) were also analyzed. $* p<0.05$, statistically significant between the indicated factors.

regard, Esler et al. showed the presence of a high level of activation of renal sympathetic outflow in patients with untreated essential HT [14], suggesting that renal sympathetic activation is substantially related to the pathogenesis of HT. Regarding the clinical significance of the sympathetic nervous system as assessed by urinary CAs, Missouris et al. showed an interrelationship between urinary NA levels and blood pressure in patients with essential HT [15]. In the present study, it was found that changes of HR were correlated with the urinary ratio of NA/DA. Given the finding that urinary NA excretion and resting HR are increased in relation to the severity of HT [15], these findings imply that sympathetic overactivity may play an important role in the etiology and progression of essential HT.
As for the interrelationships between urinary levels of CAs and glucose tolerance, Chamapaneri et al. showed that men, but not women, with diabetes had significantly lower urinary levels of CAs than those in men without diabetes among the various neuroendocrine parameters they examined [16]. The present study also showed that patients with preexisting diabetes (hemoglobin A1c $\geq 6.5$ ) had significantly higher levels of urinary NA excretion. Also, the levels of blood glucose were correlated to the levels of urinary NA and the ratios of $\mathrm{AD} / \mathrm{NA}$. It is thus thought that the pathophysiology of diabetes is, at least in part, associated with neuroendocrine dysregulation, though there might be some differences depending on the situations and gender.

In view of the relationship between urinary CAs and 

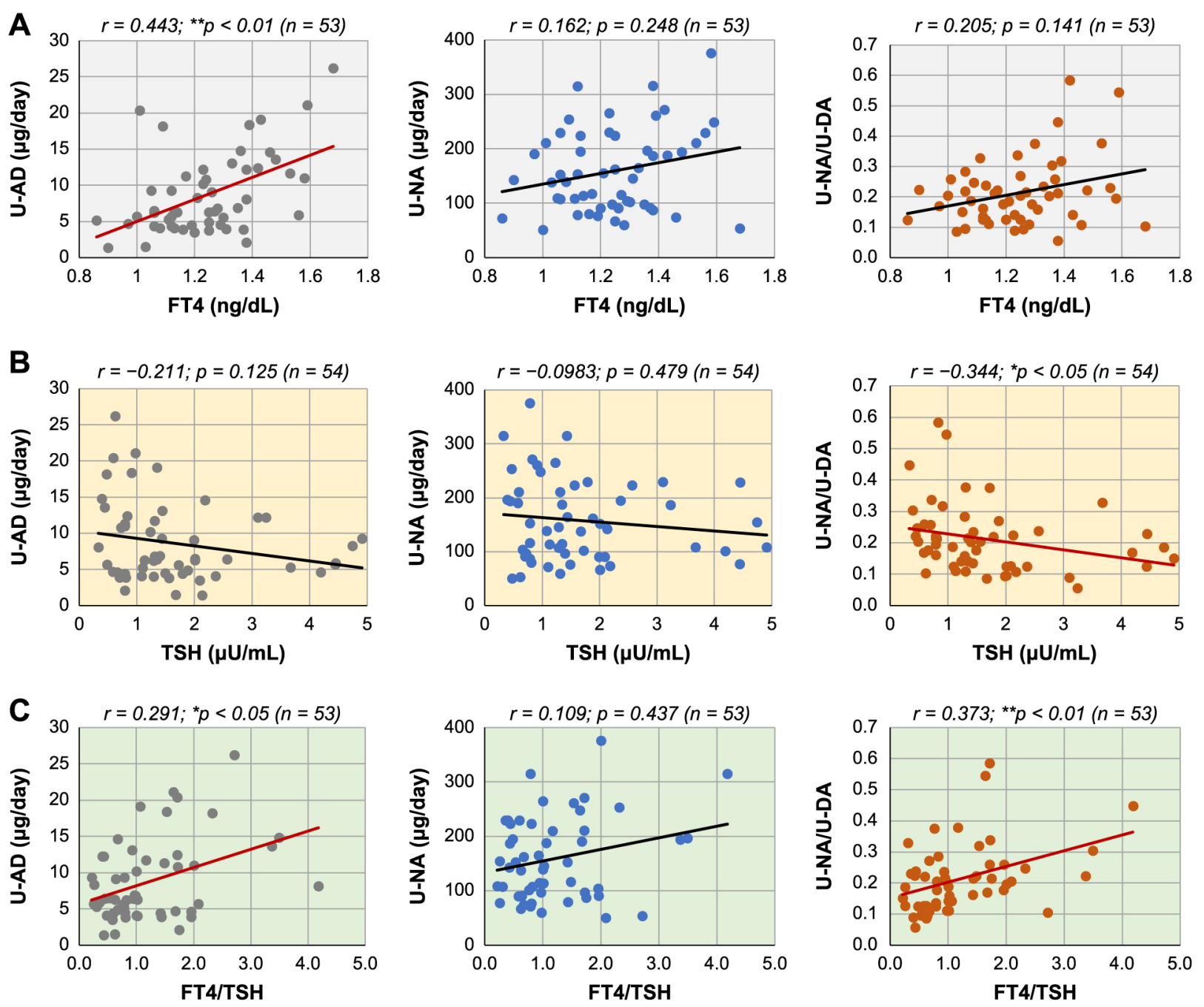

Fig. 4 Interrelationships between thyroid functions and urinary levels of catecholamines. The patients were further examined for interrelationships between urinary levels of catecholamines (CAs), including adrenaline (AD) and noradrenaline (NA), or ratios of urinary $\mathrm{AD} / \mathrm{NA}$ and NA/DA and levels of serum free thyroxine (FT4; A) and thyrotropin (TSH; B) and ratios of FT4/TSH (C). $* * p<0.01$ and $* p<0.05$, statistically significant between the indicated factors.

metabolic activity, Hollstein et al. demonstrated a functional link between CAs and energy expenditure [17]. They showed that NA rather than AD was independently associated with both energy expenditure and sleeping metabolic rate. Thus, sympathetic nervous system activity, mediated via NA, is one of the determinants of human energy expenditure in a basal non-stress condition [17]. In the present study, it was revealed that increases of HR are related to the enhancement of urinary NA and that increased thyroid hormones are also involved in enhanced conversion from DA to NA. Taken together, the results indicate that the systemic level of NA might be a useful marker to know the basal metabolic status related to susceptibility to metabolic syndrome.

The mutual interaction between the levels of urinary CAs and thyroid hormone levels may be involved in some critical disorders. For instance, thyroid hormones enable sensitization of the heart for CAs by stimulating $\beta 1$ receptor expression in cardiomyocytes [18, 19]. An excess of thyroid hormones might potentiate the effects of CAs in cardiovascular tissues, leading to increased sensitivity to stress events, which might be involved in the etiology of Takotsubo cardiomyopathy [19]. The results of our study may also indicate the presence of a functional sympatho-adrenergic system in various patients who visit a general medicine department. Unfortunately, since the sample number for FT3 measurement was much smaller than that for FT4 measurement in this retrospective study, a prospective study for assessment of the interrelationships between levels of FT3 and CAs is needed.

In the present study, there was no significant difference on urinary DA levels between the groups based on 
various clinical background. However, the sympathorenal system including local DA contents could also be related to the pathophysiology of arterial hypertension and cardiovascular damages [20]. On the other hand, sympathetic neural factors including NA and $\mathrm{AD}$ are potentially involved in the pathophysiology of lifethreatening cardiac disorders, especially in patients with heart failure, obesity, sleep apnea, or CKD [21, 22], which are closely related to marked activation of sympathetic stimuli [23].

For instance, both NA and AD levels were elevated in plasma and urine in obstructive sleep apnea patients [24, 25]. Animal studies also suggested that intermittent hypoxia facilitates catecholamine secretion from the adrenal medulla [25]. Persistent sympathetic activation and hypertension associated with sleep apnea are functionally linked to sympathetic activation and action of elevated circulating levels of $\mathrm{CA}$ on the vasculature. Hence, it is important to take care of latent lifethreatening conditions accelerated by sympathetic neural activities that are detected by a urinary NA excess.

Collectively, the results of the present study revealed that urinary NA excretion and/or the conversion of DA to NA are increased in patients with histories of HT and diabetes. Increases of HR, but not blood pressure, are linked to enhancement of urinary NA excretion, and increased thyroid hormones are also involved in the increases in urinary $\mathrm{AD}$ levels and the conversion of DA to NA. On the other hand, when a decision is made to measure urinary CAs, the patient's history of lifestylerelated diseases and the patient's HR and thyroid functions should also be taken into account for evaluating activity of the sympathetic nervous system in the clinical setting of general practice.

In the present study, the conversion steps from DA to $\mathrm{NA}$ and from NA to $\mathrm{AD}$ were analyzed, for the first time, as urinary ratios of $\mathrm{AD} / \mathrm{NA}$ and NA/DA in addition to the levels of urinary CAs. However, there were several limitations in the present cross-sectional study. Urinary CA levels might be affected by various foods, stress conditions, and ingredients such as caffeine, nicotine, and amine-rich foods [26], although the sampling conditions were carefully performed in each patient in our study. Urinary CA levels can be affected by medications including antihypertensive drugs and tricyclic antidepressant drugs, and it has also been reported that urinary NA excretion can be increased in hypertensive patients taking single-drug therapy with either a longacting dihydropyridine calcium antagonist or a $\beta$-blocker [15].

Serum levels of FT3 in a larger number of patients should also be analyzed to determine the direct interaction between thyroid hormones and CAs, though there is some difficulty for measuring FT3 under the coverage of Japanese medical insurance. In addition, more specific and sensitive methods such as LC-MS/MS for quantitative determination of urinary CAs would be ideal to exclude the effects of structurally related drugs and metabolites [27]. Finally, this study was performed as a retrospective single-center study in which sample sizes were relatively small and blood samplings were not always performed in the same periods. Further studies including a multi-center and prospective study in ageand gender-matched cohorts would be ideal to clarify the effectiveness of measurement of urinary CAs.

\section{Acknowledgements}

We are sincerely grateful to the clinical staff at the Department of General Medicine who contributed to the present work.

\section{Author Contributions}

F.O. and Y.H. conceived and designed the study; N.S., Y.H., K.Y. and Y.N. performed data collection; T.N., H.H. and K.H. analyzed the data; and N.S. and F.O. wrote and H.H. revised the paper. All authors have read and agreed to publish the final version of the manuscript.

\section{Disclosure}

The authors have nothing to disclose.

\section{References}

1. Kimura N, Takekoshi K, Naruse M (2018) Risk stratification on pheochromocytoma and paraganglioma from laboratory and clinical medicine. J Clin Med 7: 242.

2. Otsuka F, Ogura T, Nakagawa M, Hayakawa N, Kataoka $\mathrm{H}$, et al. (1996) Normotensive bilateral pheochromocytoma with Lindau disease: case report. Endocr J 43: 719723.
3. Otsuka F, Miyoshi T, Murakami K, Inagaki K, Takeda M, et al. (2005) An extra-adrenal abdominal pheochromocytoma causing ectopic ACTH syndrome. Am J Hypertens 18: 1364-1368.

4. Nishimura Y, Yasuda M, Hasegawa K, Otsuka F (2019) A case of juvenile hypertension suggestive of adrenomedullary hyperplasia. Intern Med 58: 311. 
5. Corssmit EP, Romijn JA (2014) Clinical management of paragangliomas. Eur J Endocrinol 171: R231-R243.

6. Kano Y, Otsuka F, Takeda M, Suzuki J, Inagaki K, et al. (2005) Regulatory roles of bone morphogenetic proteins and glucocorticoids in catecholamine production by rat pheochromocytoma cells. Endocrinology 146: 5332-5340.

7. Komatsubara M, Hara T, Hosoya T, Toma K, TsukamotoYamauchi N, et al. (2017) Melatonin regulates catecholamine biosynthesis by modulating bone morphogenetic protein and glucocorticoid actions. J Steroid Biochem Mol Biol 165: 182-189.

8. Goto J, Otsuka F, Yamashita M, Suzuki J, Otani H, et al. (2009) Enhancement of aldosterone-induced catecholamine production by bone morphogenetic protein- 4 through activating Rho and SAPK/JNK pathway in adrenomedullar cells. Am J Physiol Endocrinol Metab 296: E904E916.

9. Nagatsu T, Levitt M, Udenfriend S (1964) Tyrosine hydroxylase. The initial step in norepinephrine biosynthesis. J Biol Chem 239: 2910-2917.

10. Lenders JW, Pacak K, Walther MM, Linehan WM, Mannelli M, et al. (2002) Biochemical diagnosis of pheochromocytoma: which test is best? JAMA 287: 14271434.

11. Umemura S, Arima H, Arima S, Asayama K, Dohi Y, et al. (2019) The Japanese Society of Hypertension Guidelines for the Management of Hypertension (JSH 2019). Hypertens Res 42: 1235-1481.

12. Eugster PJ, Centeno C, Dunand M, Seghezzi C, Grouzmann E (2021) Stabilization of urinary biogenic amines measured in clinical chemistry laboratories. Clin Chim Acta 514: 24-28.

13. Kanda Y (2013) Investigation of the freely available easyto-use software 'EZR' for medical statistics. Bone Marrow Transplant 48: 452-458.

14. Grassi G, Mark A, Esler M (2015) The sympathetic nervous system alterations in human hypertension. Circ Res 116: 976-990.

15. Missouris CG, Markandu ND, He FJ, Papavasileiou MV, Sever P, et al. (2016) Urinary catecholamines and the relationship with blood pressure and pharmacological therapy. J Hypertens 34: 704-709.

16. Champaneri $\mathrm{S}, \mathrm{Xu} \mathrm{X}$, Carnethon MR, Bertoni AG, Seeman T, et al. (2012) Diurnal salivary cortisol and urinary catecholamines are associated with diabetes mellitus: the Multi-Ethnic Study of Atherosclerosis. Metabolism 61: 986-995.

17. Hollstein T, Basolo A, Ando T, Votruba SB, Krakoff J, et al. (2020) Urinary norepinephrine is a metabolic determinant of 24-hour energy expenditure and sleeping metabolic rate in adult humans. J Clin Endocrinol Metab 105: $1145-1156$.

18. Bahouth SW (1991) Thyroid hormones transcriptionally regulate the beta 1-adrenergic receptor gene in cultured ventricular myocytes. J Biol Chem 266: 15863-15869.

19. Aweimer A, El-Battrawy I, Akin I, Borggrefe M, Mügge A, et al. (2021) Abnormal thyroid function is common in takotsubo syndrome and depends on two distinct mechanisms: results of a multicentre observational study. $J$ Intern Med 289: 675-687.

20. Grisk O, Rettig R (2004) Interactions between the sympathetic nervous system and the kidneys in arterial hypertension. Cardiovasc Res 61: 238-246.

21. Shen MJ, Zipes DP (2014) Role of the autonomic nervous system in modulating cardiac arrhythmias. Circ Res 114: 1004-1021.

22. Kang SW (2013) Adrenergic genetic mechanisms in hypertension and hypertensive kidney disease. Electrolyte Blood Press 11: 24-28.

23. Grassi G, Seravalle G, Cattaneo BM, Lanfranchi A, Vailati $\mathrm{S}$, et al. (1995) Sympathetic activation and loss of reflex sympathetic control in mild congestive heart failure. Circulation 92: 3206-3211.

24. Makino S, Iwata M, Fujiwara M, Ike S, Tateyama H (2006) A case of sleep apnea syndrome manifesting severe hypertension with high plasma norepinephrine levels. Endocr J 53: 363-369.

25. Prabhakar NR, Kumar GK (2010) Mechanisms of sympathetic activation and blood pressure elevation by intermittent hypoxia. Respir Physiol Neurobiol 174: 156-161.

26. Lenders JW, Duh QY, Eisenhofer G, Gimenez-Roqueplo AP, Grebe SK, et al. (2014) Pheochromocytoma and paraganglioma: an endocrine society clinical practice guideline. J Clin Endocrinol Metab 99: 1915-1942.

27. Li XS, Li S, Wynveen P, Mork K, Kellermann G (2014) Development and validation of a specific and sensitive LC-MS/MS method for quantification of urinary catecholamines and application in biological variation studies. Anal Bioanal Chem 406: 7287-7297. 